\title{
DULSITA, ANG KABUUAN NG KONTRADIKSYON NG IMAHEN NI SHARON CUNETA SA PELIKULANG FILIPINO
}

\author{
Jerrick Josue David \\ University of the Philippines Film Institute \\ jheck.david@gmail.com
}

\begin{abstract}
Rolando B. Tolentino described Sharon Cuneta as the "perpetual virgin" of Philippine movies. In his essay titled "Si Sharon Cuneta at ang Perpetwal na Birhen" [Sharon Cuneta and the Perpetual Virgin], Tolentino described the perpetual virgin as eternally "meek, subservient, patient, the proverbial model of kindness and/or goodness." Such characteristic is exemplified via the typical role Cuneta plays onscreen: the good-hearted woman who chooses to forgive her oppressor(s) despite the hardships she endured in their hands. Offscreen, she seems to epitomize such an image: loving and dutiful daughter to her parents, martyr wife to her philandering first husband, devoted wife who opted to forsake a thriving career for the sake of her second husband, and doting mother to her children. Though there seems to be some truth to this imaging, such characterization feels limited, constricted, and outdated. It is one-dimensional and dismisses distinct individual characteristics. Upon closer examination, Sharon is not a perpetual virgin for she deviates from such behavior from time to time. She is not always as meek as the proverbial lamb inasmuch as the tigress in her emerges when push comes to shove. She is not totally subservient for there are moments when she rebels against accustomed expectations. She is not ever-patient for there are limitations to what she can willingly take. Moreover, she is not immaculately well-behaved for she can be rough and feisty in certain situations. Like any other human being, there are contradictions present in Sharon's character. These details were initially suppressed in the media in order to conform to the virgin image, or had gone unnoticed because of the presence of the dominant (virginal) behavior. These contradictions, when duly acknowledged, give Sharon the breadth of her being. In her movies, Sharon is generally kind and long-suffering but she can be brutal when needed. The writer of this article coined for such a paradoxical characteristic the term dulsita, a coflation of two Spanish words namely dulce (which means sweet) and maldita (which means feisty). Sharon's modesty, thoughtfulness, warmth, and charm encompass her sweet nature while her boldness, aggression, and rebellion comprise her pugnacious side, both of which may now be found in the roles she portrays in movies. She may appear weak and docile at first but she will eventually stand on her own and find the strength to fight
\end{abstract}


back. She may suffer and wallow in silence for the meantime but her voice will soon be heard. When faced with her enemies, she remains graceful and poised. Using her wit and quick tongue, she lashes out at them unknowingly. Offscreen, Sharon is no different. She has an overall charming persona that can become unwelcoming to undeserving people. She may be forgiving but she won't allow insults and baseless accusations hurled at her and her family. She may be a normally happy person but there are moments when grief gets the best of her. These contradictions shape Sharon's personality and entire being. In her so-far 37 years in the industry, she has characteristically changed and evolved as an artist. Once dubbed as the "poor little rich girl," where contradictions are present as well, she has portrayed varied roles of women in different times and various conditions: from daughter to mother, from wife to mistress, from sophisticated to uncouth, from martyr to strong-willed woman, from virtuous to wicked. The presence of oppositions is not meant to confuse or mislead her audience but rather suggests that they embrace the differences of a more honest and direct self-portrait of Sharon Cuneta in and out of movies.

\section{Keywords}

komiks, melodrama, perpetual virgin, star persona, unruly women

\section{About the Author}

Jerrick Josue David has attended trainings and seminars in film and television production under GMA Artist Center and ABS-CBN Film Productions, Inc. (Star Cinema). As an independent filmmaker and critic, his works have been recognized by GMA Network at the Sindie Film Festival in 2006 and Cultural Center of the Philippines during the 24th Gawad CCP Para sa Sining in 2012. He is currently a master's candidate at the University of the Philippines Film Institute, working on his thesis on Sharon Cuneta, after which he plans to use his star-text specialization to study other Filipino celebrities' star images. 
Perpetwal na birhen, ganito itinuring ni Rolando B. Tolentino si Sharon Cuneta sa pelikula sa kanyang sanaysay na "Sharon Cuneta at ang Perpetwal na Birhen." Ang perpetwal na birhen ay may katangiang "maamo, sunudsunuran, matiisin, ang proberbiyal na mabait at mabuti" (Tolentino 63). Hindi lamang ito nasasalin sa mga pelikula kundi maging sa iba't ibang aspekto ng buhay showbiz ni Sharon: telebisyon, musika, magasin, adbertisment. Maging ang kanyang aktuwal na persona ay sinasabi ni Tolentino na sumasalamin sa ganitong uri ng representasyon (70): siya na kumakatawan sa birheng babae sa loob ng pelikula at siya bilang babaeng birhen sa labas ng pelikula (67). Ang pagiging babaeng birhen niya sa aktuwal na realidad ang siyang nakapagpapatawid ng kanyang naratibo bilang birheng babae sa pelikula na tila tinatanggap at hindi kinukwestiyon ng manonood. Naisasakahon siya sa isang partikular na karakter dahil ito ang inaakalang papel niya sa buhay: mapagmahal at masunuring anak sa kanyang mga namayapang magulang; martir sa dating nangaliwang asawa na si Gabby Concepcion; butihing asawa na iniwan ang namamayagpag na karera upang suportahan ang pangalawang asawang si Francis Pangilinan nang mag-aral ito sa ibang bansa; at maaruga at supportive na ina sa kanyang mga anak katulad ni KC Concepcion na ngayon ay kabilang na sa industriya. Ang kanyang mga karakter ay hindi lumalayo sa kung paano siya tinitingnan ng kanyang mga tagahanga at manonood. "[Tanging] ang karakter at personalidad lamang ni Sharon ang makapagpapalusot ng naratibo ng mabait at mabuti," pagbibigay-diin ni Tolentino (63). Kung ano ang kanilang napanonood sa sine ang siya ring tila nakikita nilang ginagampanan ni Sharon sa kanyang buhay sa labas nito.

Upang maisakatuparan ang birheng karakter, mapapaloob ito sa isang pormula ng naratibo na paulit-ulit gagamitin upang patuloy na tangkilikin ng mga tagasubabay ni Sharon. Ang balangkas ng kanyang naratibo ang magsisilbing gabay kung paano ito isusulat at magdidikta sa kalalabasan ng pelikula. Makikita sa balangkas ng naratibo ang sumusunod: "1) inaapi, 2) tutulungan ng isang taong bigla na lamang sumulpot, 3) yayaman-sisikat, 4) maghihiganti, at 5) magpapatawad" (67). Dagdag pa sa pormula ay ang lalaki bilang sentro ng atensiyon at kaligayahan ng birhen (67). Iinog ang kanyang mundo sa naturang lalaki na siyang magiging dahilan ng kanyang mga pagdaraanan sa buhay.

Ang susunod na bahagi ay magpopokus sa aspektong pelikula ng sanaysay ni Tolentino. Susuriin nito ang karamihan sa mga pelikula ni Sharon upang patotohanan at pahindian ang turan ni Tolentino sa kanya. May basehan ba ang sinasabi ni Tolentino na karakterisasyon at daloy ng kuwento sa mga pelikula ni Sharon? Hindi maikakailang may hawig ang ilan (o maaaring karamihan) sa kanila subalit hindi ito sapat upang tawaging perpetwal na birhen si Sharon sapagkat may mga pagkakataong bumabalikwas siya sa nasabing turan. Makitid at limitado itong pagtingin dahil hindi pinakikita ang kanyang kabuuang pagkatao. Mayroong mga 
ugaling isinasantabi upang bigyang-diin ang birheng imahen o kaya ay may mga ugaling hindi napapansin subalit naroroon [present] pabor sa mas nakaangat na ugali. Pinaglipasan na rin ito ng panahon sapagkat makailang ulit nang lumabas si Sharon sa mga nakasanayan niyang karakter sa pelikula. Sa loob ng 34 na taon sa pelikula, hindi nanatili sa kahong pinaglalagakan ang kanyang karakter. Nagkaroon ito ng iba't ibang pagbabago, pag-aangkop at ebolusyon na masasabing tatak Sharon Cuneta.

\section{ANG BIRHEN NG KOMIKS AT MELODRAMA}

Sa pagsusuri ng karamihan sa mga pelikula ni Sharon, iilan lamang ang masasabing sumusunod sa istraktura ng naratibong nabanggit ni Tolentino. Hindi malinaw sa kanyang paglalahad kung mahigpit ba itong sinusunod at kailangang palooban ng lahat ng elementong binanggit niya. Kung sakali bang may kulang, ito pa rin ba ang sinasabing pormula o nagbago na ba ang anyo nito?

Kung susundin ang naturang balangkas at magiging mahigpit ukol dito, mula sa 53 na pelikula ni Sharon, maaari lamang itong iangkop sa kanyang limang pelikula: Dapat Ka Bang Mahalin? (dir. Borlaza, 1984), Bukas Luluhod ang mga Tala (dir. Borlaza, 1984), Bituing Walang Ningning (dir. Borlaza, 1985), Pasan Ko ang Daigdig (dir. Brocka, 1987), at Babangon Ako't Dudurugin Kita (dir. Brocka, 1989). Pinapalooban ang mga ito ng mga elementong binanggit ni Tolentino at pinagdaanan ng mga karakter ni Sharon.

Ang balangkas ng naratibo na sinasabi ni Tolentino ay ang sumusunod:
1) inaapi,
2) tutulungan ng isang taong bigla na lamang sumulpot,
3) yayaman-sisikat,
4) maghihiganti, at
5) magpapatawad. (67)

Sa Dapat Ka Bang Mahalin? bilang Myrna, magmumula ang kanyang pangaapi sa asawang si Lito na hindi pa handang talikuran ang pagbubuhay-binata kapalit ng kanilang maagang pag-aasawa. Sa Bukas Luluhod ang mga Tala bilang Rebecca, aalipustahin siya ng mayaman at legal na pamilya ng amang tinalikuran ang responsibilidad sa kanya bilang tunay na ama. Sa Bituing Walang Ningning bilang Dorina, mamaliitin siya ng mismong idolo na hindi matatanggap ang untiunti niyang pagsikat. Sa Pasan Ko ang Daigdig bilang Lupe, ikahihiya niya ang sarili dahil sa nakagisnang hirap simula nang siya ay isilang. Sa Babangon Ako't 
Dudurugin Kita bilang Salve, ipagagahasa siya ng asawang hindi nagmahal sa kanya nang lubos upang gamiting dahilan ng kanilang paghihiwalay (see Figure 1).
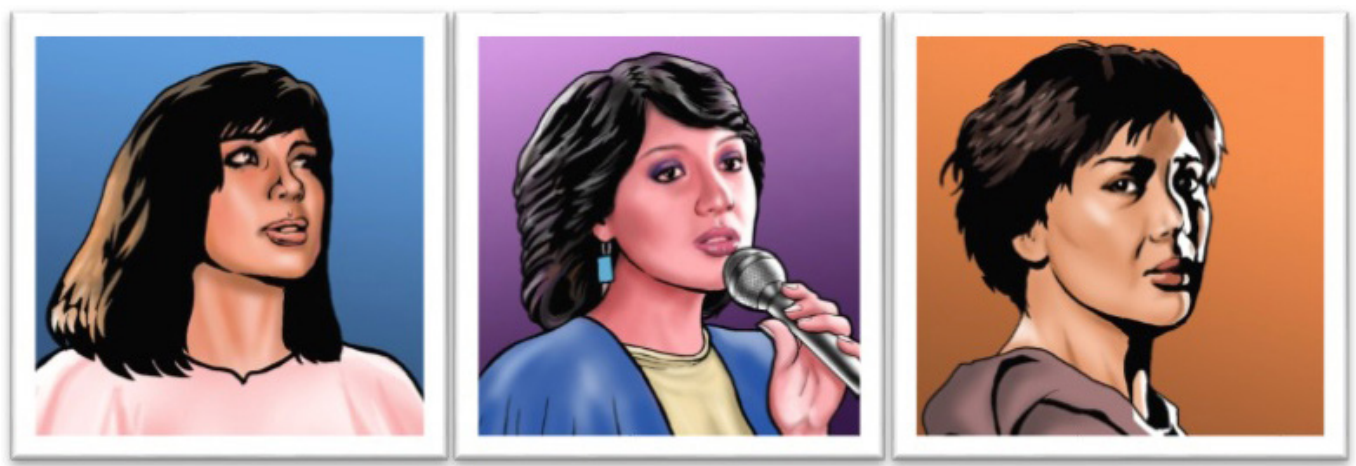

Fig. 1. Mula kaliwa: Rebecca sa Bukas Luluhod ang mga Tala (dir. Borlaza, 1984); Dorina sa Bituing Walang Ningning (dir. Borlaza, 1985); Lupe sa Pasan Ko ang Daigdig (dir. Brocka, 1987). Guhit ni Ernest Caritativo, ginamit nang may pahintulot.

Titiisin ni Sharon ang kalupitan hanggang may isang karakter na makatutuklas ng kanyang angking talino o galing. Tutulungan siya nitong maiahon ang sarili at makamit ang tagumpay na kanyang magiging sandata laban sa mga umaalipusta sa kanya. Sa kanyang pagyaman at/o pagsikat, mas mahihigitan niya ang kayamanang materyal ng kontrabida. Magkakaroon siya ng pagbabagong pisikal at pag-iisip na magsasakatuparan sa ninanais na paghihiganti. Subalit dahil sa taglay na kabutihangloob, pipiliin niyang magpatawad. Sa Dapat Ka Bang Mahalin?, patatawarin niya at makikipagbalikan siya sa napariwarang asawa na nangangakong magiging responsableng asawa at ama. Sa Bukas Luluhod ang mga Tala, tutulungan niya ang pamilya ng amang naghihirap na at makikipagkasundo sa kanila. Sa Bituing Walang Ningning, pipiliin niyang lisanin ang kislap ng entablado at ibabalik sa idolo. Sa Pasan Ko ang Daigdig, bibitiwan niya ang yaman at magbabalik sa pinanggalingan kapiling ang mga taong nagmamahal sa kanya. Sa Babangon Ako't Dudurugin Kita, palalayain niya ang asawang yumurak sa kanyang pagkatao nang sa gayon ay mapalaya rin ang sarili.

Kung ituturing pangkalahatang pagtingin [generalization] at magiging mahigpit sa naturang balangkas, hindi ito maaaring iangkop sa karamihan ng mga pelikula ni Sharon. Partikular lamang ito sa limang pelikulang nabanggit at siyam na porsyento ng kabuuang bilang ng mga pelikula ni Sharon. Ang mga nasabi pang mga pelikula ay hindi nabanggit ni Tolentino sa kanyang sanaysay bilang patotoo sa kanyang pahayag. Ang mga halimbawang kanyang ginamit ay Una Kang Naging Akin (dir. Guillen, 1991) at Pangako Sa 'Yo (dir. Marcos, 1992) na hindi kakikitaan ng istrakturang kanyang binalangkas. Hindi rin masasabing eksklusibo ang pormula na binuo sa mga pelikula ni Sharon upang maisakatuparan ang birheng babae. Ang 
mga pelikulang ito ay materyal na mula sa komiks. Ang komiks ay may sariling balangkas at pormang sinusundan.

Ang komiks ay isang form ng literaturang popular. Bilang isang form, hindi ito maaaring baguhin ayon kay Ronald Carballo, dating publicist ni Sharon, manunulat, at direktor (personal interview, 2014). Ang bawat elementong nakapaloob dito na tinangkilik ng kanyang tagasubaybay ay nararapat na kabilang sa paglalapat nito sa sine upang magpatuloy ang kanilang identipikasyon dito. Kung paano nila ito nabasa at sinubaybayan sa papel ay ganoon din nila ito mapapanood sa pinilakang tabing.

Ang komiks ay tinalakay ni Emmanuel A. Reyes sa isang seksiyon ng kanyang libro na "Notes on Philippine Cinema" (71-78). Predictable ang takbo ng kuwento at kadalasan ay nakaangkla sa gagawin ng bida upang malagpasan ang problema: episodic imbes na plot-oriented. May pagsusukdulan (dichotomization) ang karakterisasyon at may mga nagtutunggaling oposisyon (opposites): lalaki-babae; matanda-bata; maganda-pangit; mayaman-mahirap; mabuti-masama; bidakontrabida; birhen-puta. Nagtutunggali ang oposisyon upang mapanatili ang isa (mabuti) o magkaroon ng pagkakasundo o kompromiso ang dalawang naguumpugang panig na papabor sa mabuti (Velasco, "Komiks on Television" 129). Dahil sa limitadaong espasyo ng komiks, nagiging de-kahon o two-dimensional ang mga karakter upang madaling maintindihan ng mambabasa. Ang ganitong pagsasakahon (stereotyping) ang paraang ginamit noong silent era ng pelikula upang madaling makilala ang kaibahan ng bawat karakter sa kuwento at maiwasan ang pagkalito sa kanila.

Nag-uugnay rin ang form ng komiks sa genre na kanyang ginagamit. Iba-iba ang genre sa komiks: aksiyon, drama, komedya, romansa, horror, pantasya, erotika. Melodrama ang mga materyal na isinapelikula ni Sharon na kilala sa tawag na "komiks movies" (Reyes 72). Sa Hollywood, "woman's picture" ang tawag dito. Babae ang pinagtutuunang manonood nito at mga babae rin ang bida. Kung hindi sa pag-ahon sa hirap ng bida umiikot ang kuwento, ang pagkamit o pagkawala ng lalaking minamahal ang magiging sentro ng kanyang pagsubok. Ang madalas niyang karibal sa lalaki ay ang kontrabidang babae na may ugaling taliwas sa kanya. Kung birhen ang bida, ang kontrabida ay ang puta.

Samakatuwid, ang sinasabing pagiging birheng babae ni Sharon at ang naratibong pumapaloob dito ay hindi galing sa kanyang mga pelikula kundi sa form (komiks) at genre (melodrama) na pinagmulan nito. Hindi sila nabuo at binuo para kay Sharon. Inilapat sila para kay Sharon nang isapelikula niya ang mga naturang materyal. May umiiral nang ganitong karakter at naratibo bago pa niya gawin ito. Sa genre pa lamang ay may tiyak na maasahan na ang mambabasa. Kapag 
kuwentong romansa, maaasahan nilang magkakatuluyan ang bidang babae at bidang lalaki kahit may mga balakid sa kanilang pagmamahalan. Kapag melodrama, mapagtatagumpayan ng babaeng bida ang pang-aalipustang matatanggap sa mga kontrabida. Maaaring si Sharon ay naging representasyon ng birhen sapagkat ilang beses niyang ginampanan ang papel na ito. Subalit si Sharon lamang ba ang matatawag na birhen sa pelikula? Maaari nga bang ipaangkin sa kanya ang katawagang "perpetwal na birhen"?

\section{ANG BIRHEN}

Sinasabi ni Tolentino na "tanging ang karakter at personalidad lamang ni Sharon ang makapagpapalusot ng naratibo ng mabait at mabuti" (Tolentino 63). Siya sa papel ng birheng babae sa pagkatao ng babaeng birhen. Hindi maaaring tanggapin ang ganitong proposisyon sapagkat maraming nagdaang bidang babae sa pelikula ang naging mukha ng birhen. Mayroon silang katangiang birhen na kanilang naging imahen sa mga panahong namayagpag ang kanilang pangalan sa industriya. Ang bawat dekada ay nagkaroon ng kanya-kanyang representasyon ng birhen.

Sa pag-aaral ni Cesar D. Orsal, ang katulad nina Gloria Romero noong '5os, Susan Roces noong '6os, Nora Aunor noong '70s, Sharon Cuneta noong '80s, at Judy Ann Santos noong '9os ang kumatawan sa pagiging birhen sa pelikula at industriya. Hindi tahasang itinuring ni Orsal na birhen ang mga nasabing babae subalit sa kanyang paglalarawan, sumasalamin sila sa mga karakter na mabait at mabuti lalo na sa mga unang taon nila sa industriya. Nailuklok sila sa pedestal dahil siniguro ng mga nangangasiwa sa kanilang karera na magiging representasyon sila ng tinatawag na "ideyal": huwaran sa pagiging mabuting tao at iniidolo dahil sa kanilang kagandahang taglay at kapuri-puring kilos (see Figure 2).
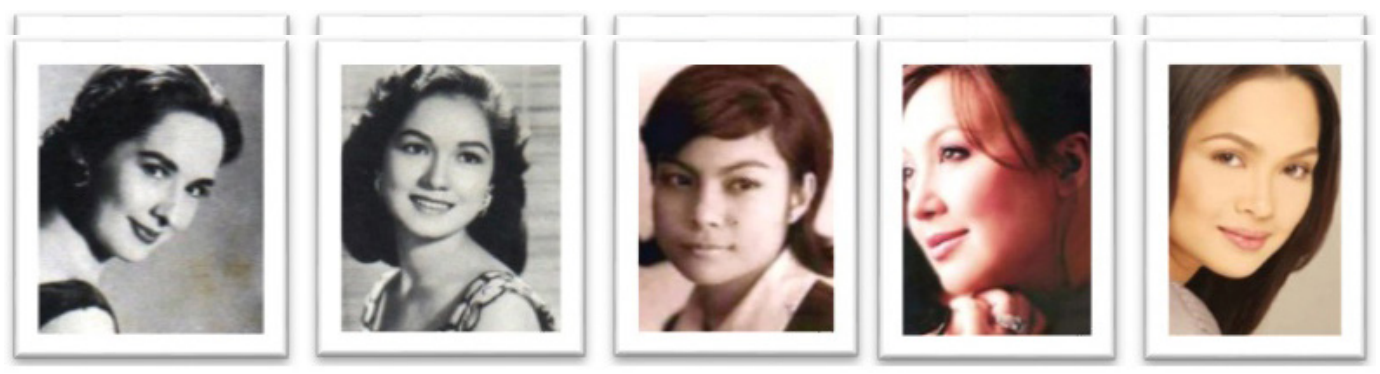

Fig. 2. Mula kaliwa: Publicity shots nina Gloria Romero, Susan Roces, Nora Aunor, Sharon Cuneta, at Judy Ann Santos. 
Si Gloria Romero, "nang matuklasan ni Dr. Jose R. Perez ng Sampaguita Pictures...noong 1948, inihulma na siya bilang arketipong artista na ikakahon sa imahe ng babaeng birhen," ayon kay Orsal (68). Sa katunayan, labing-anim na taon siyang gumanap sa papel ni Birheng Maria sapagkat itinuring ang kanyang maamong mukha at mayuming gawi na nalalapit kay Maria. Bukod pa rito, "alagad ng Diyos, masunuring anak, at masakripisyong mangingibig" (29) ang madalas niyang ginagampanang papel. Si Susan Roces ay tiningnan bilang isang "ulirang babae: huwaran ng malinis na imahe at bituin" (36) na kanyang napanindigan nang maging butihing asawa ni Fernando Poe, Jr. Nailagay siya sa trono ng reyna nang mapangasawa ang kinikilalang hari ng pelikulang Filipino. Si Nora Aunor ang naging mukha ng kaapihan (20) na pilit gagawa ng paraan upang makatakas sa kaapihang dinaranas. Hindi niya nanaising kalabanin o paghigantihan ang mga taong nang-aapi sa kanya. Ang layunin niya ay makalayo sa kanila upang gumaan ang buhay. Siya ang idolo ng masa at ng karaniwang tao. Siya ang sumalamin sa maaari nilang mapagtagumpayan sa buhay. Si Judy Ann ang larawan "ng mga babaeng inaapi at dinudusta ngunit sa bandang huli'y napapanaig ang kabutihan upang angkinin ang nararapat para sa kanya" (118). Bagaman karakterisasyon sa telebisyon ang nabanggit ni Orsal, nasalin din ni Judy Ann ang papel na ito sa pelikula. Lumaki siya sa mata ng publiko at nakita ang kanyang bawat hinaing, dusa, pangarap. Nalalaman nila na ang susi sa kanyang tagumpay ay ang angking kabutihang loob.

Nakaangkla sa umpisa ang imaheng birhen ng mga bituin sa taglay nilang maaamong mukha at mahinhing kilos subalit kinalaunan, katulad ni Gloria, pinangatawanan na ang imahen sa simpleng postura at galaw dahil "nakundisyon (na) ang produksiyon at ang mga manonood" (Orsal 69). Sa kaso ni Sharon, madalas siyang simple at ordinaryong asawa kumpara sa mga kontrabidang babae na agresibo at glamorosa (Tolentino 66). Nanatili ang kanyang wholesome na imahen maging sa iba't ibang larangan ng industriya kaya nasangkot man sa video (sex) scandal at diumano'y drug abuse, hindi pinaniwalaan ng publiko (68). Napaiigting pa ang imahen kung patuloy itong ginagampanan at paulit-ulit na nakikita ng mga manonood. "By offering a series of similar roles, the studio could unify the onscreen image of a star," ani McDonald (6o). May mga pagkakataong nanatili ang kanilang pagkabirhen sa gitna ng madilim na mundong ginagalawan ng karakter nila na maaaring bentaha o desbentaha sa kanilang pagganap. Si Susan, nanatiling inosente kahit naglublob sa mundo ng mga kriminal sa Mga Ligaw na Bulaklak (dir. Tony Cayado, 1957). Si Nora, hindi naging masama sa kabila ng mapait na dinanas sa loob at labas ng piitan sa Bulaklak sa City Jail (dir. Mario O' Hara, 1984). Si Sharon, nangibabaw ang kabutihang-loob nang patawarin niya ang asawang umalipusta sa kanya sa Babangon Ako't Dudurugin Kita (dir. Brocka, 1989). Si Judy Ann, hindi epektibong nailarawan ang papel ni Sabel (dir. Joel Lamangan, 2004) na dumaan sa karimlan ng buhay sa paghahanap ng sarili sapagkat nahirapan siyang iwaksi ang 
kabirhenan subalit sa Mga Mumunting Lihim (dir. Jose Javier Reyes, 2012), nagamit niya ito upang maikubli ang kanyang pagmamanipula sa mga kaibigan.

Kaakibat sa pagpapanatili sa imahen ang "morality clause" sa kontrata na maninigurong wasto at mabuti ang gawi at kilos ng mga bituin sa loob at labas ng pelikula (Velasco, "Packaging and Imaging for a Profitable Market Position" 77). Paliwanag ni McDonald, "the representation of a wholesome off-screen existence achieved moral closure between the star's on-screen and off-screen existence" (31). Tumutulong dito ang kontroladong publisidad ng mga istudyo (Velasco, "Packaging and Imaging" 77) o ang personal na relasyon ng mga bituin sa mga manunulat na nagkukubli ng kanilang "sikreto" tulad ng bisyo sa likod ng kamera. Si Susan Roces, hindi hinayaang makita ng kanyang mga tagahanga o kaya ay makunan ng larawan ang kanyang paninigarilyo (Velasco, "Packaging and Imaging" 77). Gayon din si Sharon na tinago ang paninigarilyo noon upang hindi pamarisan ng iba (Gulle 163). Si Nora Aunor, pag-inom ng alak ang solusyon sa kabang nararamdaman bago lumabas ng entablado.

Sa madaling salita, hindi nararapat sabihing "tanging ang karakter at personalidad lamang ni Sharon ang makapagpapalusot ng naratibo ng mabait at mabuti" (Tolentino 63) dahil may nauna sa kanya at may sumunod pa. Hindi siya natatangi dahil pare-pareho nilang pinagdaanan ang pagiging babaeng birhen. Representasyon sila ng kani-kanilang henerasyon ng birheng babae. Sila ang nagpuno sa pangangailangan ng dekadang kanilang kinabibilangan.

Si Gloria, mula sa dekada singkwenta, ang isa sa mga ginawang ehemplo ng kagandahan at kalinisan sa mga manonood na patuloy na bumabangon sa pinsalang dulot ng digmaan. Si Susan na naging tagapagmana ng trono ng idolo niyang si Gloria noong dekada sisenta ang binansagang mukha ng "pag-asa at kaliwanagan" (Orsal 34) sa panahong nag-aaklas ang mga tauhan sa loob ng industriya. Si Nora ang naging reyna ng masa noong dekada sitenta nang basagin niya ang pamamayagpag ng mga mestisa sa industriya na kaakibat ng pag-aasam ng sambayanan na magkaroon ng pagbabago sa pamahalaan. Si Sharon ang naging mukha ng bagong kabataan na bagaman sumusunod sa takbo ng panahon ay nananatiling may tradisyonal na ugali at gawi. Siya ang pumagitna sa aktibismo at liberalismo sa pelikula (at lipunan) na tila nagpahupa sa dulot nitong ingay. Si Judy Ann ang reyna ng telebisyon na tumawid sa pelikula noong dekada nobenta. Namayagpag siya sa panahong nagbago ang landscape ng industriya na mula pelikula ay telebisyon ang naging pangunahing libangan ng masang kinabibilangan ng mga kabataan, maybahay, at kasambahay. Siya ang mukha ng pagiging ordinaryo sa panahon ng teknolohiya at globalisasyon (Tolentino 106). May iba pang mga artistang sumailalim sa ganitong karakterisasyon subalit pinili ni Orsal ang mga 
nabanggit base sa kanilang impluwensiya sa publiko, hatak sa takilya, at lawak ng mga tagahanga.

\section{ANG MALDITA}

Karaniwan sa pelikula at industriya ang pagsusukdulan upang magkaroon ng pagkakakilanlan. Kadalasang hinahanay sa pagbibida o sa pagkokontrabida ang isang artista. Sa ganitong paghihiwalay, nagkakaroon ng katauhan ang mga karakter na hindi magkakatulad. Binibilang sila sa kung ano ang tama o mali at hinihiwalay sa kung ano ang mabuti o masama.

Karaniwan din sa industriya ang pagtatapat ng mga bidang babae sa pelikula. Bilang isang komodity, mayroon silang natatanging imahen upang pumatok sa kani-kanilang tagapagtangkilik. Sisiguruhin ng prodyuser na ang isang bida ay naiiba sa katapat niyang bida. Ang katapat ng bidang birhen ay ang bidang maldita.

Ang katapat ng mga bidang birhen, ayon pa rin kay Orsal, ay sina Nida Blanca noong '50s, Amalia Fuentes noong '6os, Vilma Santos noong '7os, Maricel Soriano noong '8os, at Claudine Barretto noong '9os. (Si Claudine ay hindi nabanggit ni Orsal sa kanyang pag-aaral subalit binilang ko siya sa listahan sapagkat magkatapat sila ni Judy Ann sa kanilang panahon.) Pinagdaanan din nila ang pagiging birhen sa kanilang pagsisimula subalit piniling lumihis ng daan kinalaunan. Isa itong paraan upang ibahin ang sarili sa katapat na bida na naging daan upang magampanang sabay ang pagiging bida at kontrabida habang ang bidang birhen ay naikahon sa iisang arketipo. Mas malawak ang mga papel na kanilang nilalarawan sapagkat malaya nilang pinahahayag ang sarili sa paraang ninanais (see Figure 3).
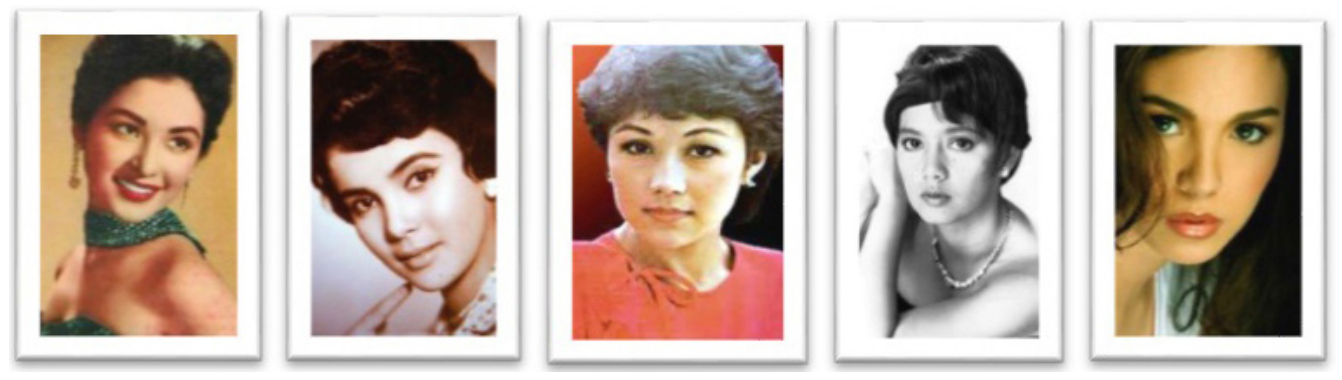

Fig. 3. Mula kaliwa: Publicity shots nina Nida Blanca, Amalia Fuentes, Vilma Santos, Maricel Soriano, Claudine Barretto.

Si Nida Blanca ang kinilalang "orihinal na mataray na bituin" (Orsal 32). Hindi siya nangiming ipagtanggol ang sarili sa sinumang nanlalamang sa kanya o sa kanyang kapwa. "Tomboyish" siyang kumilos na salungat sa pagiging "prim and 
proper" ni Gloria; matapang at walang inuurungan na kabaligtaran ng pagiging matiisin at masakripisyo ni Gloria. Si Amalia Fuentes ay may kaisipang hindi maaaring panghawakan ninuman. Pinahahayag niya ang nilalaman ng isipan na walang pakundangan sa maaaring masagasaan. Kaiba sa sinasabing pagiging "tahimik at madiplomasya" (39) ni Susan Roces. Nang magmatyur si Vilma Santos, unti-unti niyang hinubad ang kahinhinan kapalit ng mga mapangahas na papel. Gumanap siya sa mga karakter na may malawak at makabagong pagtingin sa buhay. Bida-kontrabida ang turing sa kanya sa pagsasabuhay ng papel ng mga babaeng matapang at ginagamit ang katawan at pagkababae sa pakikipagsapalaran sa buhay. Siya ang "larawan ng modernong babae na nakawala sa mga tradisyong kinagisnan" (20). Si Maricel Soriano ang naging tagapagmana ng pag-uugali at pagkilos na pinakita ng mga nauna sa kanya. "Taray Queen” ang tawag sa kanya ng industriya katulad ni Nida. Malaya niyang sinasabi ang nasa isipan at hindi nagdadalawang-isip kuwestiyunin ang mga pangyayari sa paligid kagaya ni Amalia. Mga bida-kontrabida rin ang mga naging papel niya kapareho ni Vilma. Hindi siya nagpapailalim sa dikta ng lalaki at lipunan, at naninindigan siya sa kanyang katwiran. Si Claudine Barretto, sa kabila ng kanyang maamong mukha at kahinhinan, may nagkukubling babaeng agresibo, palaban, at suwail na ginagamit niyang pananggalang sa mga taong kumakalaban sa kanya.

Ang maldita ay matapang. Nakikipagsabayan siya sa mundo ng mga lalaki upang makausad sa buhay. Lakas-loob siyang haharap sa kanila lalo na kung siya ay tinatapakan. Kadalasan, pinag-iisa ang dalawang kasarian kagaya ni Nida na sinasabuhay ang pagiging tomboy sa kanyang ugali at asal, palaban at walang inuurungan. Noong '5os, walang-takot niyang sinampal ang aroganteng tsuper ng taksi na nakasagi sa kanyang sasakyan at walang takot na umakyat sa kabundukan upang pasukuin ang kumander ng komunista (Orsal 33). Ang kanyang pinakitang katapangan sa labas ng pelikula ang siya ring ginagampanan niya sa loob nito (73). Kung tomboy si Nida, babaeng bakla naman ang representasyon ni Maricel: "nag-aangkin ng dalawang kasarian: ang pagkababae na iniingatan ang bayolohikal na katangian ngunit may pailalim na pagsupil sa pagdodomina ng iba at ang pagkabinabae na may ugaling di papatalo, di maisasantabi ang katwiran at naipapahayag ang ibig nang walang pangingimi" (101). Mapapanood madalas ang ganitong karakter ni Maricel sa mga pelikula niyang komedya gayon din si Nida.

Ang maldita ay in touch sa kanyang pagkababae. Ginagamit niya ito sa kanyang kapakinabangan sa paraang hindi nayuyurakan ang kanyang dangal o pagkatao. Alam niya kung paano paiikutin sa kanyang palad ang mga lalaki upang makuha ang kanyang gusto. Si Amalia noong '6os ang "representasyon ng mga babaeng minamahal at kinakatakutan" dahil ang kanyang taglay na kagandahan ay may kakayahang bihagin at supilin ang kapangyarihan ng mga lalaki sa mga pelikula niyang horror (Orsal 81). Subalit tanyag man sa kanyang kagandahan, ninanais 
niyang iwang pamana ang kuwento ng kanyang tagumpay. "I love women who in their lifetime have done something about their lives other than just being beautiful," aniya (Protacio 69). Siya ang kauna-unahang female independent producer at unang tumanggap ng pinakamataas na talent fee sa kanyang henerasyon (Protacio 70). Si Vilma, sa kabilang banda, "ay naglalarawan sa matapang na babae, matatag at may makabagong pananaw sa buhay...liberated" (Orsal 91, italiko ay mula sa orihinal na pahayag). Minamaliit man ng lipunan ang mga ginagampanan niyang karakter kagaya ng isang mananayaw sa Burlesk Queen (dir. Celso Ad. Castillo, 1977), kerida sa Relasyon (dir. Ishmael Bernal, 1982), at hostess sa Alyas Baby Tsina (dir. Marilou Diaz-Abaya, 1984), hindi niya ikinakahon ang mga ito sa tipikal na paglalarawan at binibigyan ng pantaong dimensiyon [human dimension]. Pinahahalagahan niya ang kanilang karanasan at hindi inaalisan ng dignidad bilang tao at bilang isang babae.

Ang pagiging maldita ay hindi nangangahulugang negatibo at lubos na taliwas sa ugali ng birhen. Bida pa rin siya kaya hindi siya maaaring magpakasama-sama nang tuluyan katulad ng kontrabidang puta subalit handa siyang magpakaputa, maitawid lamang ang pangangailangan ng pamilya kagaya ni Claudine sa Milan (dir. Olivia Lamasan, 2004). Nagagawa niya ang mga bagay na hindi inaasahan sa mga birhen: ang maging palaban at agresibo sa loob at labas ng pelikula na kabaligtaran ng pagiging lubos na matiisin nito. Buong loob niyang ipagtatanggol ang sarili sa mga pagkakataong nalalagay siya sa alanganin. Ginagawa niya ang kanyang gusto at hindi hahayaang mapanghawakan ng kung ano mang kontrata. Mapangahas siya sa kanyang kilos at hindi nangangamba sa magiging reaksyon ng tagasubaybay dahil kabilang ito sa kanyang self-expression.

Ang ganitong pagsasatipo ng dalawang bida (birhen at maldita) ay pagkakaroon ng tiyak na pagkakaiba sa kanila na nagiging bentaha para sa kani-kanilang karera. Nagkakaroon sila ng kani-kanilang timpla na magtatangi sa kani-kanilang hinahaing gawa sa merkado at publiko.

\section{DULSITA, ANG KOMBINASYON NG BIRHEN AT MALDITA}

Ang mga artista ay hindi maaaring manahan sa kahong kanilang pinaglalagakan kung nais nilang magtagal sa industriya. Nararapat silang sumunod sa takbo at pangangailangan ng panahon, maging sa kanilang edad, upang mapanatili sa kamalayan ng publiko at maging ukol [relevant] sa panahon. Hindi naiiba ang mga bituing birhen sa pelikula na nakailang-ulit na ring iwinaksi ang naturang anyo sa iba-iba nilang pelikula. May mga panahong bumabali sila nang kaunti o kaya ay tuluyang bumabalikwas sa nakagawiang papel kagaya ni Gloria Romero na nang magbalik sa industriya noong dekada '8o, "binuwag...ang isteryutipong imahe na 
ipinatupad sa kanya" (Orsal 123). Gumanap siya bilang bungangera at lasenggang landlady sa programang Palibhasa Lalake (dir. Johnny Manahan, 1987) sa telebisyon sa loob ng labing-isang taon; bilang matapobre at sugarol na ina sa Saan Nagtatago ang Pag-ibig? (dir. Eddie Garcia, 1987) at Kapag Langit ang Humatol (dir. Laurice Guillen, 1990); at bilang lider ng mga sindikato sa Condemned (dir. Mario O'Hara, 1984). Si Nora Aunor, pinili nang iwanan ang papel ng mga babaeng api at "ginamit...ang pagkaapi upang isulong ang pagkakamit ng katarungan at ibagsak ang kapangyarihan ng lalaking nagmamanipula sa takbo ng buhay sa lipunan" (Orsal 130) sa mga pelikulang Minsa'y Isang Gamu-gamo (dir. Lupita Aquino-Kashiwahara, 1976), Andrea, Paano Ba ang Maging Isang Ina? (dir. Gil Portes, 199o), at Bakit May Kahapon Pa? (dir. Joel Lamangan, 1996). Sa ganitong paraan, nasusukat ang kanilang versatility sa pagganap ng iba't ibang karakter na magpapayabong pa ng kanilang karera. Wika ni Klaprat, "offcasting not only provided the variation to sustain audience interest, but also served to enhance the image of the star as a great performer" (qtd. in McDonald 52-53).

Hindi naiiba ang daang tinahak ni Sharon. Maraming beses na siyang kumawala sa imahen ng birhen kaya hindi siya maituturing na perpetwal. Sa katunayan, maging sa mga nabanggit na komiks movies ay may mga elementong napapaloob na hindi angkop sa sinasabing pormula at pag-uugali ng birhen. Sa Pasan Ko ang Daigdig (dir. Brocka, 1987), ginahasa si Sharon at nagtangkang parusahan ang lalaking lumapastangan sa kanya na malayo sa pagiging matiisin ng birhen. Inisip niyang ipalaglag ang nabuong bata sa sinapupunan na labag sa batas ng pagiging mabait at mabuti. Sa Babangon Ako't Dudurugin Kita (dir. Brocka, 1989), mas pinili niyang magpatawad at bigyang-laya ang nang-alipustang asawa nang sa gayon ay mapalaya na rin ang sarili. Kumawala siya sa lalaking minsa'y naging sentro ng kanyang buhay. Komiks man ang pinanggalingan ay bumabalikwas pa rin sa moldeng naghulma sa pagkatao ni Sharon sa pelikula.

Si Sharon ang isa sa naging representasyon ng birhen sa pelikula. Gayon pa man, kung susuriing mabuti ang kanyang mga karakter, hindi masasabing twodimensional ang kanilang karakterisasyon. Kagaya sa totoong buhay, may mga oposisyong namamayani na kumakatawan sa buong pagkatao at naging tatak ni Sharon: birhen ngunit may pagkamaldita; karaniwang sweet subalit may pitik na kaanghangan; ang kombinasyon ng sugar and spice.

"Dulsita" ang salitang aking gagamitin upang ilarawan si Sharon. Ito ay kombinasyon ng dalawang salitang Espanyol: 1) dulce na nangangahulugang sweet o matamis na naglalarawan sa pagiging maamo, malambing, mapagmahal, at charming ng isang Sharon sa loob at labas ng pelikula, at 2) maldita na nangangahulugang palaban [feisty] na nakikita sa pagiging matapang, agresibo, at antipatika; lumalabas sa mga panahong kinakailangang ipaglaban ang sarili at 
mga ninanais sa buhay. Ang dulsita ay may malambing na bigkas sapagkat mas namamayani pa rin ang taglay na sweetness kaysa kaanghangan.

Ganito mailalarawan ang personalidad ni Sharon sa loob at labas man ng pelikula. Si Tolentino na rin ang nagsabi na "bukas sa maraming posibilidad, sa maraming paraan ng panonood at pagbabasa" (Tolentino 78) ang teksto ni Sharon. Magmumukha mang mahina at sunud-sunuran sa umpisa, mahahanap niya ang kanyang lakas upang makatayo sa sariling mga paa at harapin ang mga nananakit sa kanya sa huli. Tahimik na magtitiis at magsasakripisyo sa una ngunit sa kalaunan, aalingawngaw ang matapang na tinig laban sa mga kaaway. Sa pagsugod sa kaaway, mananatili ang kanyang grace at poise. Hindi pisikalan ang laban. Talas ng isip at bilis ng dila ang tila isang latigong lalatay sa pagkatao ng katunggali. Makikita at maririnig ito sa mga dayalogong kanyang binibitawan sa kontrabidang babae na buong loob niyang haharapin. "Verbal joust" ang tawag dito na nagmula sa mga tradisyonal na pagtatanghal sa entablado at inilapat sa pelikula (Reyes 21). Nakikipag-espadahan ng dila ang mga kalahok upang pabagsakin ang isa't isa. "Dialogue can vary from saccharine to abusive depending on the image of the actor," paliwanag ni Reyes (21).

Sa paggawa ng pelikulang mula sa komiks nagsimulang sumuka ng maaanghang na salita si Sharon laban sa kanyang mga kaaway. Naisalin ito sa mga sumunod pa niyang pelikula kahit hindi komiks ang pinagmulan. Simple lamang ang banat, sweet pa rin kung tutuusin subalit may bahid na pang-iinsulto kagaya nang sinabi ni Myrna sa kerida ng asawa sa Dapat Ka Bang Mahalin? (dir. Borlaza, 1984): "Mabuti na lang at nakita kong matanda na pala ang kabarkada niyang babae. Dahil kung hindi, baka magselos ako." Kung minsan naman ay tahasan niyang lalapastanganin ang pagkatao ng kalaban katulad sa Kapantay ay Langit (dir. Joel Lamangan, 1994) bilang Odette: "Alam mo ba kung bakit ka iniwan ni Steve? Because you are very very sick. More than a dreaded disease. At wala pang naimbentong gamot diyan. You don't have a brain. Boba!" Sa mga panahong tinutulak siya sa pader, lumalabas ang sungay ni Sharon. Hindi siya ang nagsisimula ng away subalit sisiguruhin niyang hindi niya ito aatrasan (see Figure 4). 

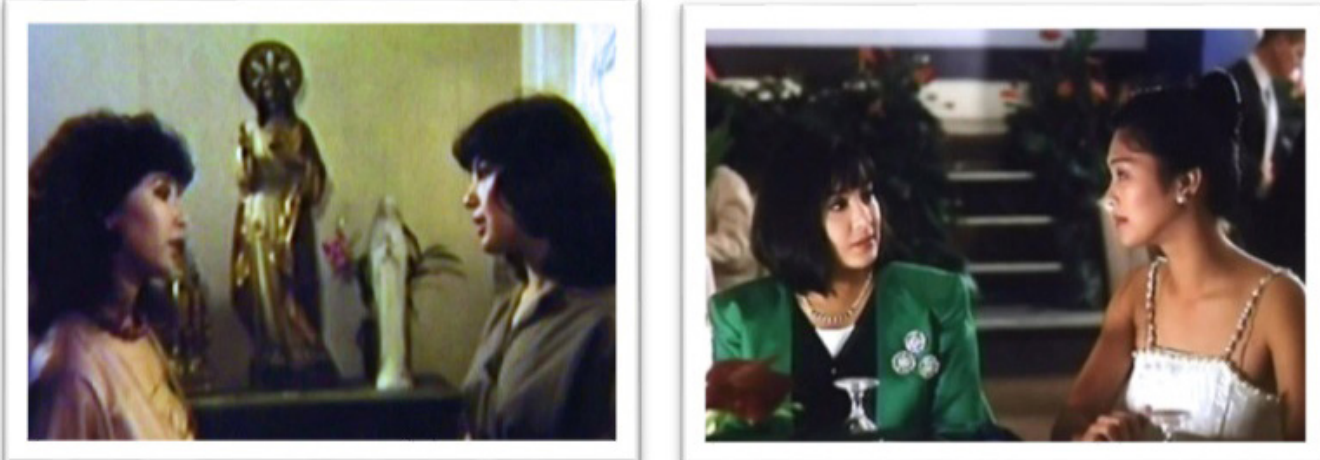

Fig. 4. Ang paghaharap ng bida at kontrabida: Dapat Ka Bang Mahalin? (dir. Emmanuel H. Borlaza, 1984), kaliwa; at Kapantay Ay Langit (dir. Joel Lamangan, 1994), kanan. Viva Films, screen captures ng may-akda.

Si Sharon ay maaari rin namang magsimula bilang maldita. Ang kanyang katarayan, katigasan, o pagmamalaki ay dulot ng mapapait na karanasan sa buhay o kaya ay simpleng pag-aangkin ng pribilehiyo ng pagiging mayaman at mataas sa lahat. Sa bandang huli, lalambot ang kanyang puso at lalabas ang taglay na kabutihang-loob. Sa Kahit Konting Pagtingin (dir. Santiago, 1990), matututong magpakumbaba si Georgia sa dating bodyguard na kanyang pinagmamataasan nang tulungan siya nito sa oras ng pangangailangan. Sa Pangako Sa 'Yo (dir. Marcos, 1992), ang kasungitan at pagtataray ni Clarissa ay malulusaw nang hayaan ang sariling umibig muli. Sa Ngayon at Kailanman (dir. Lamangan, 1992), ang katotohanan at pagpapatawad ang magpapalaya kay Aira sa pait at poot ng lumipas. Sa Kung Kailangan Mo Ako (dir. Rodriguez, 1993), ang kayabangan ni Diana ay mawawala nang matutuhan niyang pahalagahan ang ibang tao bukod sa kanyang sarili.

Si Sharon ay kadalasang "nagpapaapi dahil nagmamahal, lumalaban dahil nagmamahal, nagpapatawad dahil nagmamahal" (Tolentino 63). Ang dulsitang karakter ay hindi malayo sa birheng babae ni Tolentino subalit binibigyang-diin nito ang mga baryasyon at pag-uugaling naglalarawan ng isang buong pagkatao kaysa umiikot lamang sa iisang karakter, ang pagiging birhen. Birhen mang maituturing, hindi sila de-kahon. Birhen man ang kabuuang anyo, may ibang kulay na mas nagpapatingkad sa pagkatao nito. At sa mga panahong tinatalikuran ng birhen ang lalaking kanyang nilalayon, hindi ito nangangahulugan ng pagkawala ng kanyang katauhan kagaya ng turan ni Tolentino. Sa pagpapalaya sa lalaking sinisinta, matatagpuan niya ang kanyang sarili. 
Katumbas ng istraktura ng naratibo ni Tolentino, iaangkop ko sa isang balangkas ng naratibo ang pagpapatawid sa dulsitang karakter ni Sharon. Ito ay ang nasa Table 1.

\begin{tabular}{|l|l|}
\hline Birhen & Dulsita \\
\hline Inaapi & Iibig/umiibig \\
\hline $\begin{array}{l}\text { Tutulungan ng isang taong biglang } \\
\text { susulpot }\end{array}$ & Masasaktan/magtitiis \\
\hline Yayaman-sisikat & Tutulungan/makikilala ang sarili \\
\hline Maghihiganti & Lalaban \\
\hline Magpapatawad & Magmamahal/mamahalin \\
\hline
\end{tabular}

Table 1. Paghahambing ng Karakter ng Birhen at ng Dulsita. (Gawa ng may-akda.)

Kadalasang nasa genre ng romansa o melodrama ang mga pelikula ni Sharon kaya malaking bahagi ang lalaki sa pagdaraanan ng kanyang mga karakter. Sa pagdating ng lalaki sa kanyang buhay, iibig siya na pagmumulan ng suliranin kagaya nina April sa Dear Heart (dir. Zialcita, 1981) na unang pelikula ni Sharon at Melinda sa Mano Po 6: A Mother's Love (dir. Lamangan, 2009) na kanyang huling pelikula. Si Jimmy ay papagitna sa relasyon ni April at ng kanyang ama. Kakailanganin niyang pumili sa pagitan ng pagsunod sa kanyang puso o sa kanyang amang hindi pa siya handang pakawalan. Sa pagpasok naman ni Alfonso sa buhay ni Melinda, haharapin niya ang pang-aaping magmumula sa matapobreng pamilya nito na hindi siya tanggap bilang asawa (see Figure 5).
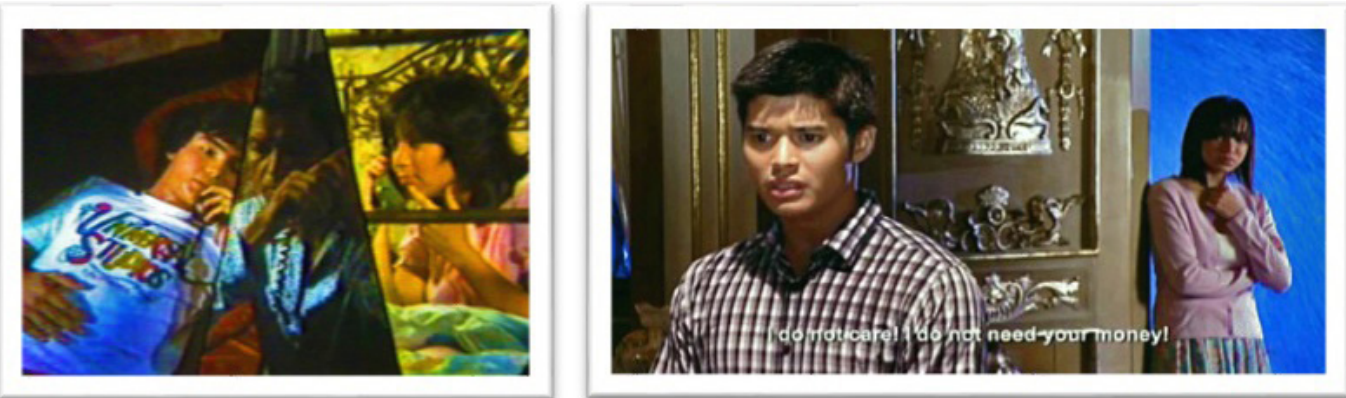

Fig. 5. Mga eksena mula sa Dear Heart (dir. Zialcita, 1981, kaliwa) at Mano Po 6: A Mother's Love (dir. Lamangan, 2009, kanan). Viva Films at Regal Films (respectively), screen captures ng may-akda.

Kung hindi man iibig, si Sharon ay umiibig na sa lalaking kanyang kasintahan o asawa. Ang pagpapatibay ng relasyon ang kanyang hangarin kahit kapalit nito ay matinding sakripisyo. Bilang si Cecile, isasantabi niya ang sariling pangangailangan upang mapagsilbihan ang asawa (Sa Hirap at Ginhawa, dir. Salvador, 1984); bilang si Elaine, kakalabanin niya pansamantala ang pamilya upang makapiling 
ang minamahal (Nakagapos na Puso, dir. Salvador, 1986); bilang si Sandra, magpapakababa at titiisin niya ang pang-aabuso ng asawa upang tuparin ang sinumpaang panata ng kasal ("Katumbas ng Kahapon" mula sa 3 Mukha ng Pag-ibig, dir. Borlaza, 1988); bilang si Diosa, ituturing niyang kerida ang sarili upang maibalik ang asawang kinasal sa iba subalit unang nagpakasal sa kanya (Una Kang Naging Akin, dir. Guillen, 1991); bilang si Mariel, titiisin niya ang malamig na pakikitungo ng stepchildren upang mapanatili ang kaayusan sa pamilya (Madrasta, dir. Lamasan, 1996); at bilang si Sarah, tatalikuran niya ang isang malaking promosyon sa trabaho upang samahang maghanapbuhay ang mister sa ibang bansa (Caregiver, dir. Roño, 2008).

Katulad ng birhen, tutulungan ang dulsita ng mga kaibigan o bagong kakilala (na kanyang magiging kaibigan) sa kanyang pinagdaraan sa buhay. Sila ang magiging ekstensiyon ng kanyang pagkatao na magmumulat sa kanyang isipan sa sitwasyong kinahaharap. Sila ang paghuhugutan niya ng lakas upang maitaguyod ang sarili. Sa kanyang pagtayo sa sariling mga paa, matututo siyang ipaglaban ang kanyang karapatan. Kaakibat ng pagbabagong kaisipan ay ang pagbabagong-anyong pisikal na magbibigay sa kanya ng kumpiyansa upang harapin ang mga taong umaapak sa kanya.

Ang dulsita ay matapang na maghahamon ng laban sa kerida ng asawa kahit siya ay nagdadalang-tao (Dapat Ka Bang Mahalin?, dir. Borlaza, 1984); susugurin niya ang babaeng lumalandi sa asawang si Arnold kahit magmukha siyang maledukado sa harapan ng marami (Sa Hirap at Ginhawa, dir. Salvador, 1984); bubuhusan niya ng alak si Lavinia sa muli nilang pagkikita bilang ganti sa pagbuhos nito sa kanya ng isang basong tubig (Bituing Walang Ningning, dir. Borlaza, 1985); lalatiguhin niya si Kadyo nang malaman niyang pinagnakawan siya nito (Pasan Ko ang Daigdig, dir. Brocka, 1987); ipadudukot at ipagagahasa niya ang kinakasama ng asawang si Alfred sa harap nito upang iparanas ang pagdurusang sinapit niya (Babangon Ako't Dudurugin Kita, dir. Brocka, 1989); makikipag-upakan at makikipagsabunutan siya sa dating kasintahan ni Tonchi upang ipagtanggol ang sarili (Tayong Dalawa, dir. Guillen, 1993); iiwanan niya ang asawang si Edward nang maagrabyado ang sariling anak sa kamay ng stepchildren (Madrasta, dir. Lamasan, 1996); isasabotahe niya ang relasyon ni Frances na itinuring na matalik na kaibigan nang malaman niyang nakikiapid ito sa kanyang asawang si Tim (BFF: Best Friends Forever, dir. Deramas, 2009); at tututukan niya ng kutsilyo at hahamunin ang hipag na hindi makatao ang trato sa kanya (Mano Po 6: A Mother's Love, dir. Lamangan, 2009).

Kapag narating na ang hangganan ng pananahimik ng dulsita, aalisin niya ang kanyang belo, huhubarin ang puting damit, at iparirinig ang kanyang tinig na may buong lakas at tapang. Hindi siya matitinag sa harap ng kaaway sapagkat hawak niya ang alas. Pipiliin din niyang magpatawad sa huli hindi lamang dahil 
sa kabutihang-loob kundi dahil bukas na ang kanyang isipan. Magmamahal na siya nang lubusan na may ganap na tiwala sa sarili at sa lalaking minamahal o, sa pagpapalaya sa lalaking minamahal, matututunan niyang mahalin ang sarili. Sa mga ganitong pagkakataon, wika ni Orsal ukol kay Sharon, "naangkin na...niya ang naratibo ng mga ekstra-ordinaryong babae na nagsisimula bilang biktima ng diskriminasyon, ngunit bumabalikwas sa sakit, obsesyon, o pag-iwas na maging alipin ng kanyang tadhana" (132).

Nang mamatay ang kanyang anak, malalaman ni Irene na hindi niya kailangan ng lalaki upang maging buo ang pagkatao kaya tinanggihan niya ang alok ng dating asawa na magsama silang muli (Kahit Wala Ka Na, dir. Borlaza, 1989) (David n.p.); nang makapaghiganti sa dating asawa, pipiliin ni Salve na pakawalan ito upang matigil na ang mismong pagpapahirap niya sa sarili (Babangon Ako't Dudurugin Kita, dir. Brocka, 1989); nang humingi ng tawad at nagnais bumalik sa kanilang pamilya ang naglokong asawa, hindi ito tatanggapin ni Amy sapagkat napagtanto niyang nabuo ang kanyang sarili nang lumisan ito (Nang Iniwan Mo Ako, dir. Reyes, 1997); nang magkaroon ng pagkakataong makasama ang lalaking nagbigayhalaga sa kanya, pipiliin ni Diane na hindi sumama alang-alang sa kanyang anak (Minsan Minahal Kita, dir. Lamasan, 20oo); nang mawalay ang anak sa kanyang piling, magsisikap si Stella na pagandahin ang kinabukasan niya at ng kanyang anak (Crying Ladies, dir. Meily, 2003); at nang piliin ng asawa na bumalik sa Pilipinas, tatanggi si Sarah dahil mas maganda ang kanyang hinaharap sa ibang bansa kaysa sa asawa (Caregiver, dir. Roño, 2006) (see Figure 6).
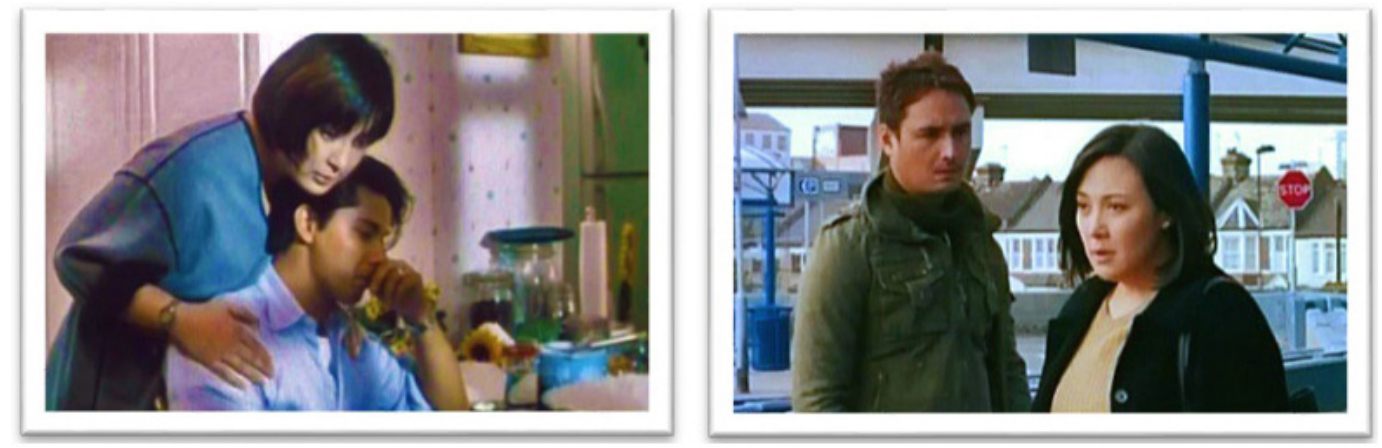

Fig. 6. Ang pag-aangkin sa sarili: Nang Iniwan Mo Ako (dir. Reyes, 1997), kaliwa; at Caregiver (dir. Roño, 2006), kanan. Viva Films and Star Cinema (respectively), screen captures ng may-akda. 


\section{SI SHARON AT ANG OPOSISYON SA KANYANG PAPEL AT PAGKATAO}

Mayaman-mahirap; maganda-pangit; mabuti-masama; payat-mataba; asawakerida; mahina-malakas. Mayamang naghirap; mahirap na yumaman; simple na naging glamorosa; masama na nagpapakabuti; mataba na nagbabawas ng timbang; asawa na naging kerida; mahina na natagpuan ang lakas. Ginampanan ni Sharon ang lahat ng ito at pinagdaanan ng kanyang mga karakter. Turan ni Orsal, “nailuklok ni Sharon ang imahe ng babae sa iba't ibang pagkakataon upang maging representasyon ng mga babae sa iba't ibang panahon" (48).

Si Sharon ang dalagang mangingibig na mauunawaang hindi sagot ang kayamanan sa kanyang kaligayahan. Siya ang breadwinner ng pamilya na magsusumikap upang makamtan ang kaginhawahan sa buhay. Siya ang asawang martir na gagamitin ang dusa at sakit upang makaipon ng lakas para lumaban at maipagtanggol ang sarili. Siya ang mapagmataas na employer na bababa sa toreng tinitirhan nang mapagtantong kailangan niya ang tulong ng kanyang manggagawa. Siya ang butihing maybahay na matagpuan ang balanse sa pagiging ina at asawa sa pagkawala ng sarili. Siya ang babaeng nakalimot sa sarili na mahahanap ang sariling kahalagahan sa kanyang pagkabigo. Ang dulsita ay may ganap na pagkatao. Ang dulsita ay husto ang pagkababae.

Sa pagsisiyasat sa kabuuang katauhan ni Sharon sa industriya, taglay niya mismo ang ganitong oposisyon. Nagmula siya sa mayamang pamilya subalit hindi matapobre. Nang ilapit siya sa masa bilang promosyon ng Pasan Ko ang Daigdig (dir. Brocka, 1987), madali niya itong nagawa sapagkat sinanay siya ng amang pulitiko na pakitunguhan ang mga taong nasa iba't ibang antas ng pamumuhay. Si Lupe ang pinakamahirap sa lahat ng mga ginampanan niyang papel dahil isa itong mangangalakal ng basura sa Smoky Mountain, marusing at mabaho. Sapantaha ng karamihan na hindi niya magagampanan ang papel subalit wala siyang arteng nagpagulong-gulong sa basura nang sumigaw na ng "Action!" si Lino Brocka. Ani Baby Gil, "The public has this belief that you have to experience something to project it onscreen. Sharon never experienced poverty so how can she project it? Ang perception ng tao, she can never project being poor on screen. Lino opposed this" (Gacoscos 28). ${ }^{2}$ Sa paglipas ng panahon at pagsusuri sa kanyang filmography, maaaring ihanay si Lupe sa mga mahuhusay niyang pagganap. Hinubad niya nang tuluyan ang pagiging elegante, nakitaan ng kagaspangan ng ugali, at piniling maging gold digger makaalis lamang sa pusaling tinitirhan na malayong-malayo sa personalidad ng birhen (see Figure 7). 

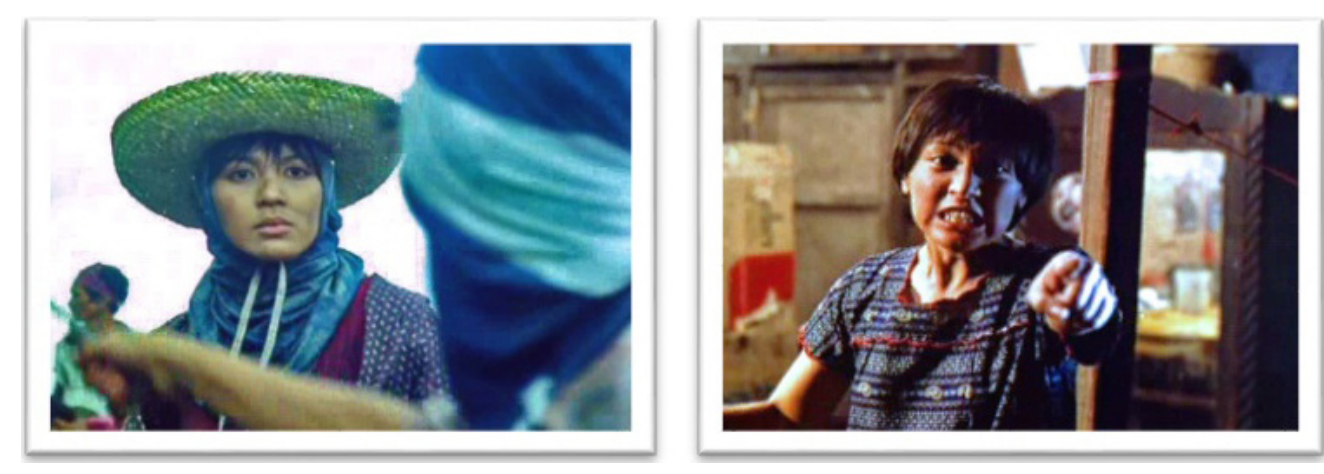

Fig. 7. Dalawang eksena mula sa Pasan Ko ang Daigdig (dir. Brocka, 1987). Viva Films, screen captures ng may-akda.

Inaamin ni Sharon na sunod siya sa layaw ngunit hindi spoiled. Sa katunayan, ang yaman ng kanyang pamilya at ang family showbiz connection ang nagtulak sa kanya upang magsikap at magtrabaho nang maigi para mapatunayan sa lahat na karapatdapat siya sa puwestong kanyang tinatamasa (Lijuaco 162). Nang makipaghiwalay siya kay Gabby, hindi siya umuwing umiiyak sa kanyang mga magulang bagkus ay nagdoble-kayod upang matustusan ang buhay nilang mag-ina kahit alam niyang hindi sila pababayaan ng kanyang mga magulang (Cruz 28). Pinanindigan niya ang pinasok niyang maagang pag-aasawa na nauwi sa hiwalayan katulad ng ilang papel na ginampanan niya sa pelikula. Sa mga sumunod niyang relasyon, bukas na ang kanyang mga mata at isipan, bitbit ang mga aral mula sa nakaraan. "I wasn't blinded by love [anymore]," aniya ("Simply KC").

Mayaman si Sharon subalit hindi karaniwang mestisa nang pasukin niya ang showbiz. Hindi niya nakuha ang Kastilaloy na katangian ng kanyang inang si Elaine Gamboa-Cuneta. Naging pabor ito sa kanya sapagkat sa pagdating ni Nora, nagtapos na rin ang pagrereyna ng mga mestisa sa industriya. "[Had] Sharon inherited those strong features, she could have been less attractive to the Pinoy multitude of the late '7os and onwards," wika ni Vibas (7).

May katabaan na si Sharon nang magsimula siya sa pelikula at telebisyon kumpara sa mga kasabayang may balingkinitang pangangatawan. Insekyur siya sa kanyang malalaking binti kaya tinago niya ang mga ito sa pagsusuot ng pantalon sa eksena sa beach sa P.S. I Love You (dir. Garcia, 1981) na sumasang-ayon din sa pinangangalagaang wholesome na imahen. Sa laki ng kanyang timbang sa ngayon, inaamin niyang dismayado siya sa sarili subalit bago pa pulaan ng iba, ginagawang biro na niya ito. Ang self-deprecating humor ang nagsisilbi niyang defense mechanism upang protektahan ang sarili laban sa mga taong nakatuon lamang sa kanyang timbang. Gayon pa man, kung bibiruin siya ng iba ukol dito, tiyak siyang masasaktan. 
Ayon sa kanyang mga kaibigan, generally sweet si Sharon subalit, pagtatapat niya, "I can be very bitchy if the situation calls for it" (Gulle 163). Katulad ng dulsita, may limitasyon ang kanyang pagtitimpi. Hindi siya patuloy na ngingiti kung harapan nang binabastos. Patunay rito ang pakikipagsagutan niya sa mga bumabatikos sa kanya at sa kanyang pamilya sa Twitter noong 2012. Nakipagbatuhan siya ng maanghang at malamang salita na kinagulat ng mga taong hindi siya lubusang kilala. Tila wala sa kanyang (birheng) karakter ang makipagbangayan (sa labas ng pelikula). Subalit para sa kanyang anak na si KC Concepcion na naging sentro ng paninirang puri, pinili niyang lumaban. "I stand up for what I think is right. I may do it the wrong way but those are my feelings," paliwanag niya ("Ang Latest"). Hindi man sang-ayon ang iba sa nagawa niyang kilos, pinaninindigan pa rin niya ang pagtatanggol sa sarili at sa anak kahit kinalaunan ay pinagsisisihan ang kawalang kontrol sa emosyon at sa hindi pagpili sa makabuluhang laban lamang (Cuneta).

Tipikal na masayahin, bungisngis, at palabiro si Sharon. "She's consistently a happy person and there are times na mapapagulong ka talaga sa sahig [sa kanyang mga biro]," paliwanag ni KC ("Simply KC"). Subalit nang tumuntong siya sa edad na apatnapu, nalugmok siya sa depresyon. Pagrerebelde ang kanyang naging tugon sa mga pagbabago sa buhay at tinapon ang pagiging propesyonal. Nawalan siya ng gana sa trabaho at nawalan ng pagpapahalaga sa mga taong nakapaligid sa kanya. "I used to be everyone's sweetheart, and then I turned forty, and I felt it all changed" (Cuneta). Sa kanyang pisikal na pagbabago, nagbago rin ang pagtanggap ng publiko sa kanya. Sa halip na yakapin at iangkop ang mga pagbabagong ito, nagpatalo siya at nagpakalunod sa kanyang inseguridad, nakalimot sa noo'y panuntunan sa buhay na, "I'm... not self-destructive.... If I fail in one area, to make myself feel good, I have to push myself para hindi ako masyadong feeling inadequate sa failure ko" (Lejano 2002). Sa kanyang matapat na pahayag kamakailan lamang, winika niya, "I felt defeated" ("The Buzz"). Gayunman, sa kanyang pagkabigo ay maasahan ang kanyang pagbangong muli.

Ang mga nabanggit na oposisyon sa katauhan ni Sharon ang nagbibigay sa kanya ng lawak sa limitadong pagkatao na nakikita at pinapakita sa pelikula at industriya. Mas malalim, mas mapanghahawakan, mas maiintindihan.

\section{LAGOM}

Ang pag-aaral sa imahen at teksto ng bituin ay puno ng kontradiksiyon. Maraming salik ang dapat isaalang-alang at mapagtugma upang makapaglabas ng isang imaheng nalalapit sa mga nagbabanggaang karakter ng bituin sa loob at labas ng pelikula. Ang pag-aaral na ito ay sinisiwalat ang mga oposisyong namamayani 
sa karakter ng bituin nang sa gayon ay mapag-isa ang mga ito at hindi manatiling oposisyon kundi isang buo at ganap na pagkatao.

Si Sharon ay unang binansagang "poor little rich girl" nang gampanan niya ang karakter ng mga dalagang sa kabila ng yaman ay hindi nakukuha ang lahat ng gusto at nakararanas ng kabiguan. Ang bansag na ito ay kakikitaan na ng oposisyon o kabalintunaan na siyang magagamit niya upang makapagpalit-palit ng anyo ayon sa hinihingi ng pagkakataon. Hindi man kasinglawak ng mga nagampanang karakter ni Maricel Soriano ang kanyang mga papel, hindi rin sila limitado. Ang kanyang pagkabirhen ay bahagi lamang ng kanyang ebolusyon na epektibo niyang nagagamit sa iba't ibang pelikula kabilang ang isa sa pinakamahalagang pelikula ng kanyang karera, ang Madrasta (dir. Lamasan, 1996). Dahil sa kanyang kabirhenan, nabago niya ang mukha ng mga madrasta na kadalasang kontrabida at nagpapahirap sa buhay ng stepchildren. Sila ay nabigyan niya ng pantaong-katangian [human qualities] na hindi nakakulong sa kanyang papel sa buhay kagaya ng ginawa ni Vilma Santos sa mga kerida sa Relasyon (dir. Ishmael Bernal, 1982). Ang madrasta ay asawa, ina, anak, apo, kapatid, boss, empleyado na binabalanse ang kanyang buhay hindi lamang sa sariling pangangailangan kundi maging sa pangangailangan ng mga tao sa kanyang paligid. Epektibo rin niyang nabago ang istilo ng kanyang pag-arte. Mula sa over-the-top acting sa ilan niyang mga pelikula, underacting style ang atake niya sa karakter na nagpaigting lalo sa pagiging matiisin ng birhen.

Wholesome man ang kabuuang pagkakakilanlan, kumakalas pa rin si Sharon sa kanyang comfort zone. Nagpagulong-gulong siya nang walang arte sa mabaho at maruming basura ng Smoky Mountain sa Pasan Ko ang Daigdig (dir. Brocka, 1987) upang magampanan nang maigi ang papel ni Lupe na sadlak at sadsad sa kahirapan ng buhay. Taliwas ito sa kadalasang maginhawang buhay ng kanyang mga karakter. Konserbatibo man sa pag-iisip at pagkilos, kakikitaan naman ng passion sa mga eksenang sekswal, wika ni Laurice Guillen nang gawin nila ang Una Kang Naging Akin noong 1991 ("Mega Tatlong Dekada"). Mula sa pagiging literal na birhen sa Kailan Sasabihing Mahal Kita (dir. Garcia, 1985) na nagsasabing, "Premarital sex is wrong," liberal siyang nakipagtalik sa lalaking hindi niya asawa at agresibong pumaibabaw rito sa Tayong Dalawa (dir. Guillen, 1993). Mula sa kadalasang pagiging unang asawa na hinihiwalayan ng lalaki, nangahas siyang maging kerida sa Minsan, Minahal Kita (dir. Lamasan, 2001). Mula sa babaeng umiinog ang mundo sa lalaki, siya ang babaeng hindi tinanggap ang nagbabalik na asawa sa Nang Iniwan Mo Ako (dir. Reyes, 1997) at iniwan ang asawang may mahinang gulugod at disposisyon sa Caregiver (dir. Roño, 2008). Sa Caregiver, sunud-sunuran man siya sa asawa (noong umpisa), mas masipag at nakaaangat siya pagdating sa trabaho. Mula sa paggawa ng mabuti, isa siyang ex-convict sa Crying Ladies (dir. Meily, 2003) na nanggagantso ng kapwa upang maitawid ang buhay sa araw-araw. Pipilitin niyang ituwid ang kanyang buhay alang-alang sa anak na nawalay sa kanya sa pamamagitan 
ng paghahanap ng trabaho bilang mang-aawit gamit ang sintunadong boses. Itinuturing ito ng kritikong si Oggs Cruz na isang dekonstruksyon sa kanyang mga naunang papel sa pelikula (Cruz n.p.): ang kadalasang may malinis na reputasyon ay isa nang manloloko; ang kadalasang nagtatagumpay sa kanyang pag-awit ay hindi matanggap-tanggap bilang mang-aawit sa Japan; ang kadalasang pinagmumulan ng hinanakit ng magulang ang siya namang magpapakabuti upang hindi maging hinanakit ng anak. Maging ang luhang madaling tumutulo sa mga madramang eksena ang siyang gagamitin niya para sa "pekeng" pagdadalamhati sa namatay (see Figure 8).
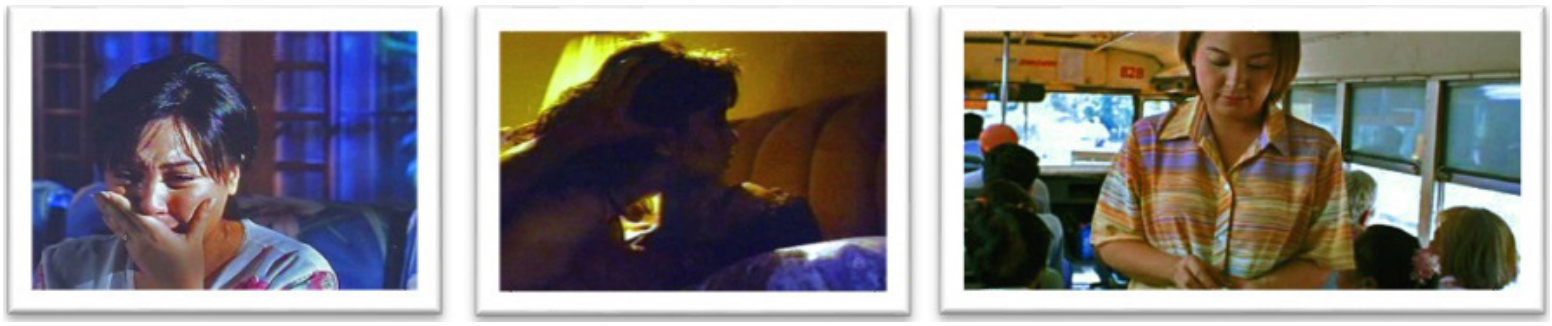

Fig. 8. Mga eksena mula sa kaliwa: Madrasta (dir. Lamasan, 1996); Tayong Dalawa (dir. Guillen, 1993); Crying Ladies (dir. Meily, 2003). Star Cinema, Viva Films, at Unitel Pictures (ayon sa pagkakabanggit), screen captures ng may-akda.

Madaling mahalintulad ang itsura ni Sharon sa karaniwang tao subalit hindi siya maituturing na karaniwang tao. Katulad ng karaniwang tao, pinaghirapan niya ang kanyang narating sa industriya subalit hindi mararating ng karaniwang tao ang kanyang posisyon anumang hirap ang kanilang pagdaanan. Bukas man ang kanyang kuwento sa publiko, may mga bagay pa rin siyang tinatago para sa kanyang sarili bilang isang pribadong tao na hindi saklaw ng industriya. Public figure man siyang maituturing, hindi niya hahayaan ang publiko sa kanilang malayang pagbatikos sa kanyang buhay. Hindi siya mananahimik at magkikibit-balikat na tila isang birhen bagkus ay ipagtatanggol niya ang sarili laban sa mga umaalipusta sa kanya. Handa rin siyang putulin ang relasyon sa mga tinuturing na malalapit na kaibigan kung tingin niya ay ginagamit lamang siya o ang kanyang estado sa buhay.

Hindi maaaring ituring na perpetwal na birhen si Sharon sa pelikula sapagkat sa likod ng kanyang sweet persona ay isang malditang nagpupumiglas sa oras na mabigyan ng pagkakataon. Hindi siya palagiang maamong tupa dahil may pagkakataong lumalabas ang kanyang pagkatigre sa panahong nalalagay siya sa alanganin. Hindi ganap ang kanyang pagiging sunud-sunuran sapagkat may mga panahong nagrerebelde siya o kumikilos na hindi ayon sa nakagawiang tradisyon. Hindi siya maikakahon sa pagiging birhen sapagkat nanlalaban at pilit na kumakawala ang maldita sa kanya. Ang birhen at maldita ay nagsasanib sa katauhan ni Sharon upang maging isa, buo, at totoong tao, sa loob o labas man ng pelikula. 


\section{Mga Tala}

1. Ang papel na ito ay kasalukuyang pinag-aaralan at sinasaliksik pa lamang bilang bahagi ng kabuuang graduate thesis ng may-akda. Si Sharon Cuneta ay hindi lamang isang bituin kundi isang higanteng bituin sa pelikulang Filipino na may pambihirang kasaysayan. Isa sa mga layunin ng pag-aaral na ito ang matukoy ang mga naging kontribusyon niya sa industriya na nagtanghal sa kanya bilang Megastar. Ang dulsitang karakter ang maituturing na kabuuan ng mga pinagdaanang imahen ni Sharon sa industriya sa loob ng 37 na taon na kanyang naging tatak at pagkakakilanlan. Ang kanyang naging ebolusyon na magpapatibay sa kanyang nabuong imahen ay patutunayan at pag-uugnayin hindi lamang sa pamamagitan ng pelikula kundi maging sa iba't ibang larangang kinabibilangan ni Sharon katulad ng musika, telebisyon, adbertisment, at pahayagan upang mas maging angkop at komprehensibo. Ang papel na ito ang isa sa mga hakbang tungo sa pagtatapos ng nasabing pag-aaral.

Malugod na pinasasalamatan ng may-akda ang mga sumusunod sa kanilang tulong, suporta, at inspirasyon sa pagbuo ng papel na ito: Bono Olgado, Joel David, Ed Lejano, Shirley Evidente, Joni Gutierrez, Rolando B. Tolentino, Cesar D. Orsal, Christian Tablazon, Gil Mariano Razon, Emma Louisa Malabanan, Ernest Caritativo, Ralph Rollin Montoya, Bong Asiao, Carlo Maghirang, Rene Torres, Marc Cabagnot, Rodney Bantog, Maria Lourdes Cabero, Tina Sharonian, Angelo de Guzman, Ronald Carballo, Ed de Leon, Mauro Feria Tumbocon, Jr., Oggs Cruz, All Things Sharon (Facebook group page), Sharonians, at Sharon Cuneta.

2. Sa panulat ni Nestor U. Torre noong 2008, pinahayag niya ang naging pribadong usapan nila ni Lino Brocka ukol sa pagganap ni Sharon sa Pasan Ko ang Daigdig: "Privately, Brocka later expressed his frustration to us over his inability to help Sharon understand the thoughts and feelings of a very destitute person. $\mathrm{He}$ lauded her for wanting to become a better actress, but fretted that her 'sheltered' existence made it difficult for her to empathize with and fully understand 'poor' characters." Maging si Torre ay naniniwalang hindi angkop ang proyekto para kay Sharon. 


\section{Mga Sanggunian}

Allen, Robert C., and Douglas Gomery. Film History: Theory and Practice. New York: McGraw-Hill, 1985. Print.

Borlaza, Emmanuel H., dir. Bituing Walang Ningning. Scr. Orlando R. Nadres. Perf. Sharon Cuneta, Cherie Gil, Christopher de Leon, Joel Torre, Jay Ilagan, Tommy Abuel, Chanda Romero, Lorli Villanueva. Viva, 1985. Film.

- _ , dir. Bukas Luluhod ang mga Tala. Scr. Jose Carreon. Perf. Sharon Cuneta, Gina Pareño, Eddie Rodriguez, Pilar Pilapil, Tommy Abuel, Rey "PJ" Abellana, Raymond Lauchengco, Lani Mercado, Eula Valdez, Manny Castañeda. Viva, 1984. Film.

- - , dir. Dapat Ka Bang Mahalin? Scr. Raquel Villavicencio. Perf. Sharon Cuneta, Gabby Concepcion, Chanda Romero, Vic Silayan, Celia Rodriguez, Rez Cortez, Robert Campos, Chito Ponce Enrile, Mon Alvir. Viva, 1984. Film.

-_-, dir. Kahit Wala Ka Na. Scr. Jose Javier Reyes. Perf. Sharon Cuneta, Richard Gomez, Tonton Gutierrez, Cherie Gil, Simon Soler, Mila del Sol, Chanda Romero, Tommy Abuel, Joonee Gamboa, Tita Muñoz, Mia Gutierrez, Rina Reyes. Viva, 1989. Film.

_- , dir. "Katumbas ng Kahapon." Mula sa omnibus na 3 Mukha ng Pag-ibig. Scr. Jose Javier Reyes. Perf. Sharon Cuneta, Christopher de Leon, Mat Ranillo III, Subas Herrero, Rosemarie Gil, Suzanne Gonzales, Zandro Zamora, Eddie Arenas. Viva, 1988. Film.

Brocka, Lino, dir. Babangon Ako't Dudurugin Kita. Scr. Jun Chionglo. Perf. SharonCuneta, Hilda Koronel, Christopher de Leon, Bembol Roco, Mona Lisa, Ruben Rustia, William Lorenzo. Viva, 1989. Film.

- - , dir. Pasan Ko ang Daigdig. Scr. Rene O. Villanueva. Perf. Sharon Cuneta, Loretta Marquez, Tonton Gutierrez, Raoul Aragon, Princess Punzalan, Mario Montenegro, Mark Gil, Deborah Sun, Anita Linda, Rey "PJ” Abellana, Marilen Martinez. Viva, 1987. Film.

The Buzz. Sharon Cuneta, int. Boy Abunda. ABS-CBN. Quezon City, 2015. Television.

Carballo, Ronald. Personal interview. Quezon City, 2014.

Cruz, Edgar O. "Megastar, Megabucks." Star Studio (Nov. 2002): 28-29. Print. 
Cruz, Oggs. "Re: Crying Ladies." Pinoydvd.com, 12 Jan. 2004. Web. 31 Mar. 2014.

Cuneta, Sharon. "Sharon Cuneta's Open Letter to Her Public." All Things Sharon, Aug. 19, 2014. Web. June 20, 2015.

David, Jerrick J. "Ang Asawa at ang Kerida sa Kahit Wala Ka Na (Borlaza, 1989)." All Things Sharon, 9 Apr. 2013. Web. 25 Apr. 2015.

Deramas, Wenn, dir. BFF: Best Friends Forever. Scr. Mel Mendoza-del Rosario. Perf. Sharon Cuneta, Aiai delas Alas, John Estrada, Gina Pareño, Chokoleit, Joy Viado, Empress Shuck, Miles Ocampo, Nash Aguas, DJ Durano. Star, 2009. Film.

Dyer, Richard. Heavenly Bodies: Film Stars and Society. London: Macmillan Education, 1987. Print.

- Stars. London: BFI, 1992. Print.

Gacoscos, Blaise. "Sharon.” Yes! (Jan.-Feb. 2003): 22-52. Print.

Garcia, Eddie, dir. Kailan Sasabihing Mahal Kita. Scr. Orlando Nadres. Perf. Sharon Cuneta, Christopher de Leon, Joel Alano, Eddie Rodriguez, Cherie Gil, Liza Lorena, Armida Siguion-Reyna, Paraluman. Viva, 1985. Film.

-_- dir. Kung Aagawin Mo ang Lahat sa Akin. Scr. Jose Javier Reyes, Gina Marissa Tagasa-Gil. Perf. Sharon Cuneta, Dante Rivero, Laurice Guillen, Jackie Lou Blanco, Ricky Davao, Tonton Gutierrez, Ali Sotto, Francis Magalona, Alicia Alonzo, Rosemarie Gil, Luis Gonzales, Vina Morales. Viva, 1987. Film.

- - , dir. P.S. I Love You. Scr. Edgar Reyes. Perf. Sharon Cuneta, Gabby Concepcion, Boots Anson-Roa, Eddie Garcia, Nova Villa, Lampel Luis, Barbara Perez, Joel Alano, Tony Carreon, Norma Japitana. Viva, 1981. Film.

Guillen, Laurice, dir. Tayong Dalawa. Scr. Jose Dalisay, Jr. Perf. Sharon Cuneta, Gabby Concepcion, Eric Quizon, Barbara Perez, Bing Loyzaga, Mary Gale Russel, Nida Blanca, Anita Linda, Mutya Crisostomo, Tony Mabesa, Augusta Victa. Viva, 1992. Film.

- - , dir. Una Kang Naging Akin. Scr. Orlando R. Nadres. Perf. Sharon Cuneta, Gabby Concepcion, Dawn Zulueta, Armida Siguion-Reyna, Charito Solis, Eddie Gutierrez, Michael de Mesa, Jinky Oda, Patrick Guzman, Alicia Alonzo, Gino Antonio. Viva, 1991. Film.

Gulle, Ramil. "Sweet Reinvention." Metro (Oct. 2007): 160-164. Print. 
Lamangan, Joel, dir. Kapantay Ay Langit. Scr. Emmanuel H. Borlaza. Perf. Sharon Cuneta, Richard Gomez, Bing Loyzaga, Charina Scott, Tonton Gutierrez, Subas Herrero, Rosemarie Gil, Hazel Huelves, Romeo Rivera, Lander Vera-Perez. Viva, 1994. Film.

-_- dir. Mano Po 6: A Mother's Love. Scr. Roy Iglesias. Perf. Sharon Cuneta, Christopher de Leon, Zsa Zsa Padilla, Heart Evangelista, Dennis Trillo, Ciara Sotto, Kris Aquino, Zoren Legaspi, Ryan Eigenman, Niña Jose, John Manalo, Nicole Uysiuseng, Glaiza de Castro, JC de Vera. Regal, 2009. Film.

-_-, dir. Ngayon at Kailanman. Scr. Orlando R. Nadres. Perf. Sharon Cuneta, Richard Gomez, Cherie Gil, Caridad Sanchez, Mark Gil, Ruben Rustia, Liza Lorena, Rustom Padilla, Mutya Crisostomo, Cecille Galvez. Viva, 1992. Film.

Lamasan, Olivia, dir. Madrasta. Scr. Ricardo Lee. Perf. Sharon Cuneta, Christopher de Leon, Claudine Barretto, Patrick Garcia, Camille Prats, Zsa Zsa Padilla, Rico Yan, Vangie Labalan, Nida Blanca, Teresa Loyzaga, Cris Villanueva, Eula Valdez, Koko Trinidad, Tita Muñoz. Star, 1996. Film.

-_- dir. Minsan, Minahal Kita. Scr. Ricky Lee. Perf. Sharon Cuneta, Richard Gomez, Edu Manzano, Angel Aquino, Carmina Villaroel, Rosemarie Gil, Ciara Sotto, January Isaac, Marvin Agustin, Kristine Hermosa, Gabe Mercado, Carl Brian V. Ravanes, Ama Quiambao. Star, 200o. Film.

Ang Latest. Sharon Cuneta, int. Jose Javier Reyes. TV5. Quezon City, 2013. Television.

Lejano, Apol. “The Megastar on Money." Good Housekeeping (Sept. 2002): 50-55. Print.

Lijuaco, Chit. "Far from the Madding Crowd." The Phippine Tatler (Dec. 2006): 156-165. Print.

Marcos, Pepe, dir. Pangako sa 'Yo. Scr. Humilde “Meek” Roxas. Perf. Sharon Cuneta, Ramon "Bong" Revilla, Jr., Caridad Sanchez, Edu Manzano, Subas Herrero, Pinky Amador, Donna Cruz, Chuckie Dreyfuss, Lucita Soriano, Manjo del Mundo, Atong Redillas, Jeffrey Veloso. Viva, 1992. Film.

McDonald, Paul. The Star System: Hollywood's Production of Popular Identities. New York: Wallflower, 2011. Print.

Mega Tatlong Dekada. Headwriter Chris Violago. ABS-CBN. Quezon City, 2008. Television. 
Meily, Mark, dir. Crying Ladies. Scr. Mark Meily, Melvin Lee, Tony Gloria, Francis Xavier Pasion, Jeane Lim, Jay Lozada. Perf. Sharon Cuneta, Angel Aquino, Hilda Koronel, Eric Quizon, Ricky Davao, Julio Pacheco, Shamaine Buencamino, Sherry Lara, Gileth Sandico, Johnny Delgado, Edgar Mortiz, Raymond Bagatsing, Lou Veloso, Joan Bitagcol. Unitel, 2005. Film.

Morin, Edgar. The Stars. Minneapolis: U of Minnesota P, 2005. Print.

Orsal, Cesar D. Movie Queen: Pagbuo ng Mito at Kapangyarihang Kultural ng Babae sa Lipunan. Quezon City: New Day, 2007. Print.

Protacio, Romy. "Amalia Fuentes: The Matriach Who Led the Muhlach Movie Dynasty." Balik

Tanaw: The Filipino Movie Stars of Yesteryears, Volume 1. San Diego: Asian Journal San

Diego, 2010. 66-73. Print.

Reyes, Emmanuel A. Notes on Philippine Cinema. Manila: De La Salle UP, 1989. Print.

Reyes, Jose Javier, dir. and scr. Nang Iniwan Mo Ako. Perf. Sharon Cuneta, Albert Martinez, Matthew Mendoza, Valentin Simon, Maritoni Fernandez, Jackie Castillejo, Dindi Gallardo, Manny Castañeda, Mandy Ochoa, Alain Sia, Eddie Arenas, Luz Valdez, Ruby Moreno, Mel Kimura. Viva, 1997. Film.

Rodriguez, Eddie, dir. Kung Kailangan Mo Ako. Scr. Ricky Lee, Humilde "Meek" Roxas, Eddie Rodriguez. Perf. Sharon Cuneta, Rudy Fernandez, Dick Israel, Miguel Rodriguez, Johnny Delgado, Caridad Sanchez, RR Herrera, Teresa Loyzaga, Christopher de Venecia, Chin-chin Rodriguez. Viva, 1993. Film.

_-_, dir. Maging Sino Ka Man. Scr. Emmanuel H. Borlaza, Eddie Rodriguez. Perf. Sharon Cuneta, Robin Padilla, Edu Manzano, Vina Morales, Dennis Padilla, Rosemarie Gil, Suzanne Gonzales, Ali Sotto, Romeo Rivera, Rez Cortez, Christopher Roxas, Guila Alvarez, Carmi Matic, Kimchi Matias, Daryl Lance Sarita. Viva, 1991. Film.

Roño, Chito, dir. Caregiver. Scr. Chris Martinez. Perf. Sharon Cuneta, John Estrada, Rica Peralejo, Jhong Hilario, Makisig Morales, John Manalo, Lotlot de Leon, Mickey Ferriols, Anita Linda, Saul Reichlin, Matthew Rutherford, Claire Jeater, Marita Zobel, Monique Wilson, Ima Castro. Star, 2008. Film.

Salvador, Leroy, dir. Nakagapos na Puso. Scr. Jose Javier Reyes, Gina Marissa Tagasa. Perf. Sharon Cuneta, Lorna Tolentino, Edu Manzano, Rosal Rosal, Amado Cortez, Dawn Zulueta, Tonton Gutierrez, Vina Morales, Sandy Andolong, Ronnie Ricketts, 
Eva Darren, Romeo Rivera, Gladys Reyes, Rose Ann Gonzales, Bella Flores, Eddie Arenas. Viva, 1986. Film.

- - , dir. Sa Hirap at Ginhawa. Scr. Jose Javier Reyes. Perf. Sharon Cuneta, Vic Silayan, Gabby Concepcion, Nida Blanca, Cherie Gil, Ronnie Ricketts, Leroy Salvador, Sandy Andolong, Marita Zobel, Romeo Rivera, Joonee Gamboa, Tony Carreon. Viva, 1984. Film.

Santiago, Pablo, dir. Kahit Konting Pagtingin. Scr. Amando Lacuesta, Jr. and Jose Bartolome. Perf. Sharon Cuneta, Fernando Poe, Jr., Bing Loyzaga, Ricky Davao, Subas Herrero, Paquito Diaz, Dencio Padilla, Ali Sotto, Rey “PJ” Abellana, Ernie Zarate, Naty Santiago, Tom Olivar. Viva, 1990. Film.

Sicam, Edmund L. "Why Sharon Sells." Star Studio (Nov. 2002): 26-27. Print.

Sharon. ABS-CBN. Quezon City, 2004. Television.

Simply KC. Sharon Cuneta, int. KC Concepcion. ABS-CBN. Quezon City, 2010. Television.

Tolentino, Rolando B. "Judy Ann Santos at ang Aura ng Ordinaryo." Richard Gomez at ang Mito ng Pagkalalake, Sharon Cuneta at ang Perpetwal na Birhen at Iba Pang Sanaysay Ukol sa Bida sa Pelikula Bilang Kultural na Texto. Pasig City: Anvil, 2000. 103-09. Print.

- - . "Sharon Cuneta at ang Perpetwal na Birhen." Richard Gomez at ang Mito ng Pagkalalake, Sharon Cuneta at ang Perpetwal na Birhen at Iba Pang Sanaysay Ukol sa Bida sa Pelikula Bilang Kultural na Texto. Pasig City: Anvil, 20oo. 63-81. Print.

Torre, Nestor U. “Caregiver: Culmination of Sharon's 'Journey." Philippine Daily Inquirer, 13 June 2008. Web. 31 Mar. 2014.

Velasco, Johven. "Komiks on Television: Recycling Pinoy Pulp Fiction." Huwaran/ Hulmahan Atbp.: The Film Writings of Johven Velasco. Ed. Joel David. Quezon City: U of the Philippines, 2009. 127-30. Print.

_-_. "Packaging and Imaging for a Profitable Market Position." Huwaran/Hulmahan Atbp.: The Film Writings of Johven Velasco. Ed. Joel David. Quezon City: U of the Philippines, 2009. 69-85. Print.

Vibas, Danny. “The Sharon Cuneta Mystique." Star Studio (Nov. 2002): 6-8. Print. 
Zialcita, Danny L., dir. and scr. Dear Heart. Perf. Sharon Cuneta, Gabby Concepcion, Liza Lorena, Eddie Garcia, Rosemarie Gil, Ronaldo Valdez, Josephine Estrada, Fred Montilla, Ting Jocson, Johnny Wilson, Virginia Montes, Suzanne Gonzales, Johnny Vicar, Edu Manzano, Imelda Ilanan. Sining Silangan, 1981. Film. 